\title{
Detrimental Effect of C-Reactive Protein on the Cardiometabolic Cells and Its Rectifying by Metabolic Surgery in Obese Diabetic Patients
}

This article was published in the following Dove Press journal: Diabetes, Metabolic Syndrome and Obesity: Targets and Therapy

\author{
Fang Sun ${ }^{1, *}$ \\ Zhigang Zhao',* \\ Qiang $\mathrm{Li}^{\prime}$ \\ Xunmei Zhou' \\ Yingsha $\mathrm{Li}^{\prime}$ \\ Hexuan Zhang' \\ Zhencheng Yan' \\ Hongbo $\mathrm{He}^{\prime}$ \\ Zhigang $\mathrm{Ke}^{2}$ \\ Yu Gao ${ }^{2}$ \\ Fan $\mathrm{Li}^{2}$ \\ Weidong Tong ${ }^{2}$ \\ Zhiming Zhu (D)' \\ 'Department of Hypertension and \\ Endocrinology, Daping Hospital, Third \\ Military Medical University, Center for \\ Hypertension and Metabolic Diseases, \\ Chongqing Institute of Hypertension, \\ Chongqing 400042, People's Republic of \\ China; ${ }^{2}$ Department of General Surgery, \\ Daping Hospital, Third Military Medical \\ University, Chongqing 400042, People's \\ Republic of China
}

*These authors contributed equally to this work
Background: High-sensitivity C-reactive protein ( $h s$-CRP) has been regarded as a biomarker of low-degree inflammation in illness; however, whether CRP exerts its pathogenic effect on the cardiometabolic system remains unknown. Aside from the beneficial effects of metabolic surgery on cardiometabolic system, its impact on inflammation still worth examining. Thus, this study aims to investigate the effect of CRP on adipose and vascular cells, and their responses to metabolic surgery in obese diabetic patients.

Patients and Methods: The expression of CRP and RAS- and ERK-related factors in the adipocytes and VSMCs were measured. Obese patients with type 2 diabetes who underwent metabolic surgery were followed up for 2 years thereafter. Laboratory tests, which included serum $h s$-CRP levels and visceral fat thickness (VFT), were obtained before and after surgery.

Results: CRP administration significantly and dose-dependently increased the intracellularfree calcium concentration $([\mathrm{Ca} 2+] \mathrm{i})$ in cultured adipocytes and in the VSMCs. CRP administration significantly increased ACE, Ang II, AT1R and p-ERK expressions, but reduced ACE2 expression in both the adipocytes and VSMCs. Clinical study showed that VFT was closely associated with serum $h s$-CRP. Furthermore, VFT and serum $h s$-CRP were found to be highly associated with blood pressure. Finally, metabolic surgery remarkably decreased blood pressure, visceral fat and serum $h s$-CRP levels.

Conclusion: CRP has a detrimental effect on cardiometabolic cells, aside from functioning merely as a biomarker. Serum $h s$-CRP levels are highly associated with hypertension and visceral obesity, which can be antagonized by metabolic surgery in obese diabetic patients. Keywords: CRP, metabolic surgery, type 2 diabetes

\section{Introduction}

Diabetes and obesity often coexist, and hypertension is twice as frequent in obese patients with type 2 diabetes compared with non-diabetic patients. ${ }^{1,2}$ Additionally, obese patients with type 2 diabetes have a greater risk of cardiovascular than nonobese diabetic individuals. ${ }^{3}$ Accordingly, obesity, diabetes, and hypertension are commonly clustered as a metabolic syndrome because of their common pathogenesis, such as insulin resistance, activation of the renin-angiotensin-aldosterone system, oxidative stress, and abnormalities of the immune system. ${ }^{4,5}$ Extensive experimental studies have shown that inflammation is closely linked with hypertension, obesity and diabetes, ${ }^{6,7}$ while clinical studies have demonstrated that obese diabetic patients have increased total leukocyte counts, which are correlated with
Correspondence: Zhiming Zhu

Tel +86-23-6876-7849

$\mathrm{Fax}+86-23-6870-5094$

Email zhuzm@yahoo.com 
insulin sensitivity and partially mediated by inflammatory changes in adipose tissue. ${ }^{8,9}$ Thus, chronic, low-grade inflammation contributes to the development of cardiometabolic diseases.

In the past decade, $h s$-CRP has been regarded as a biomarker of low-degree inflammation in illness. ${ }^{10,11}$ As a reaction protein in acute inflammation, CRP expresses in the liver and pancreas. ${ }^{12,13}$ Its synthesis and expression are regulated by activated monocytes, fibroblasts and some cytokines, with interleukin (IL)-6 and tumor necrosis factor (TNF)-alpha acting as the main stimulants of CRP synthesis and secretion. ${ }^{14,15}$ Several cross-sectional clinical trials have suggested that obesity is intimately related to the level of CRP. ${ }^{16,17}$ Moreover, serum CRP levels were related to blood pressure, blood lipids, and glucose. ${ }^{18,19}$ Baseline serum $h s$ CRP levels in healthy subjects are a good predictor for the future incidence of cardiovascular disease, stroke, sudden death and peripheral vascular diseases. ${ }^{20,21}$ Aside from the liver, adipose tissue has also been implicated in the regulation of CRP production, mediated by inflammatory factors. ${ }^{22,23}$ Although CRP is extensively applied as a biomarker of lowdegree inflammation in clinical settings, whether CRP itself participating in the pathogenesis of the cardiometabolic diseases remains unknown. The renin-angiotensin system (RAS) and extracellular signal-regulated kinases (ERK)1/2 play a crucial role in cardiovascular remodelling and dysfunction, but how CRP affects RAS and ERK 1/2 is still unclear. ${ }^{24}$

Weight reduction and the administration of hypoglycaemic and antihypertensive drugs have been shown to have anti-inflammatory effects. ${ }^{25}$ In addition, metabolic surgery has been shown to significantly improve hyperglycaemia and obesity, as well as the control of hypertension. ${ }^{26,27}$ However, it is unclear whether metabolic surgery improves cardiometabolic dysfunction through directly antagonizing inflammation. Thus, this study aims to investigate the effect of CRP on RAS and ERK in both adipose and vascular tissues, as well as the impact of metabolic surgery on blood pressure and inflammation in obese diabetic patients.

\section{Materials and Methods}

\section{Cell Culture and Treatment}

\section{Induced Differentiation of 3T3-LI Preadipocytes}

The method used for differentiation of murine 3T3-L1 adipocytes was described before. Briefly, murine 3T3-L1 cell line purchased from the Institute of Cell Biology, Shanghai Academy of Chinese Sciences. Cells were cultured in Dulbecco's modification of eagle medium (DMEM) high glucose (25 mM), 15\% fetal bovine serum (FBS), and 1\% penicillin/streptomycin. Cells incubation condition was humidified with air containing $5 \% \mathrm{CO}_{2}$. All evaluation performed on cells between 15 and 35 passages. 3T3-L1 cells, $3 \times 10^{5} /$ well, cultured in the 6 -well plate to reach $90 \%$ confluency. Then, cells differentiated into adipocytes using 3T3-L1 differentiation kit and according to the manufactures instructions. Briefly, 3T3-L1 cells culture medium was replaced with differentiation medium (DMEM/F12, 1:1) with $10 \% \mathrm{FBS}$, insulin $1.5 \mu \mathrm{g} / \mathrm{mL}$, dexamethasone $1 \mu \mathrm{M}$, IBMX $500 \mu \mathrm{M}$, and rosiglitazone $1 \mu \mathrm{M}$ and incubated for 3 days. Next, differentiation medium replaced with maintenance medium ( $1 \mu \mathrm{M}$ of insulin to $1 \mathrm{~mL}$ of DMEM/F12 (1:1) with $10 \%$ FBS). According to the manufacturer's instructions, maintenance medium changed every $2-3$ days.

\section{Primary Culture and Passage of VSMC}

Thoracic aorta was removed from anaesthetized healthy male Wistar rats (provided by the experimental animal center of Daping Hospital of Third Military Medical University), and transferred to serum-free DMEM culture medium. Medium membrane of blood vessel was separated and transferred into penicillin vial containing adequate amount of serum-free DMEM medium. The blood vessel strips were cut into $1 \mathrm{~mm} * 1 \mathrm{~mm}$ sized tissue blocks by ophthalmic scissors, and placed in $37^{\circ} \mathrm{C}$ and $5 \% \mathrm{CO}_{2}$ incubator for $3 \mathrm{~h}$ to attach to the bottom of the bottle. The cells were cultured for 4 days and then changed into fluids. The cells were fused and passaged. Cellular immunohistochemical staining with antialpha sm-actin antibody showed that the brown positive staining of intracytoplasmic myofibrils was VSMC.

\section{Cell Treatment}

The differentiated 3T3-L1 adipocytes and VSMC were divided into control group, $0.1 \mu \mathrm{g} / \mathrm{mL}$ CRP intervention group and $1 \mu \mathrm{g} / \mathrm{mL}$ CRP intervention group. After $24 \mathrm{~h}$ of intervention, the total cell protein was extracted, and the related protein expression including ACE, ACE 2, Ang II, AT1R, P-ERK, ERK, was detected by Western blotting according to the manufacturer's instructions, $\beta$-actin used for normalization. CRP mRNA in 3T3-L1 adipocytes were detected by RT-PCR according to the manufacturer's instructions, GAPDH was used for normalization. The oligonucleotides, used as PCR primers, were as follows: 5'-CTT ACG CTA CCA AGA CGA-3' and 5'-TCC CAC CAA AGA CTG ATT-3' for murine CRP, GAPDH 5'-ACG GCA AATTCA ACG GCA CAG TCA-3' and 5'-TGG GGG CAT CGG CAG AAG G-3'. 


\section{Measurement of $\mathrm{Ca}^{2+}$ Fluorescence}

The method used for measurement of $\mathrm{Ca}^{2+}$ Fluorescence was described before. 3T3-L1 adipocytes and VSMC seeded on coverslips or freshly isolated cardiac mitochondria were stained with Fura-2AM (2 mM; Sigma-Aldrich) or Rhod2AM (5 mM; Thermo Fisher Scientific) calcium fluorescent indicator for the cytosolic or cardiac mitochondrial $\mathrm{Ca}^{2+}$, respectively. The cells or mitochondria were incubated for $60 \mathrm{~min}$ at room temperature and then washed to remove the extraneous dye using a fluorescent plate reader (Fluoroskan Ascent Fluorometer; Thermo Fisher Scientific). Fura-2 fluorescence was measured using a $510 \mathrm{~nm}$ emission wavelength and 340 and $380 \mathrm{~nm}$ excitation; emission at $580 \mathrm{~nm}$ and excitation at $550 \mathrm{~nm}$ were used for Rhod-2.

\section{Immunofluorescence}

3T3-L1 preadipocytes were fixed with $4 \%$ paraformaldehyde at room temperature for $10 \mathrm{~min}$ and then bathed in $0.2 \%$ Triton X-100 for $10 \mathrm{~min}$. The cells were washed with PBS, then blocked with $10 \%$ fetal bovine serum for $30 \mathrm{~min}$ and incubated with antibodies against PKD2L1 (1:100, AB9084, rabbit; Merck), NCX1 (1:100, ab2869, mouse; Abcam), or ATP5A (1:100, ab14748, mouse; Abcam) overnight at $4{ }^{\circ} \mathrm{C}$. The cells were washed three times and then incubated with fluorescent dye-labeled secondary antibodies (Santa Cruz) at room temperature for $30 \mathrm{~min}$. DAPI was used to stain the nuclei for $5 \mathrm{~min}$. Images were acquired using a confocal microscope (LCS SP8 STED; Leica).

\section{Clinical Study \\ Patients}

In order to conduct an analysis of the relationship between CRP and multiple metabolic factors, we performed a prospective single-centre cohort trial that was in accordance with the STROBE guidelines for the presentation of clinical trials and the principles of the Declaration of Helsinki. The protocol is registered on the office site (ClinicalTrials.gov Identifier: NCT02050984). The institute's ethics committee approved this study.

A total of 127 patients who were suffering from metabolic diseases in this hospital between January 2010 and December 2018 were recruited in this study. The diagnosis of metabolic syndrome, diabetes, obesity and hypertension was based on AHA, ADA and IDF criteria. ${ }^{28-30}$ RYGB and sleeve gastrectomy were both included in the operation. The main exclusion criteria included the following: patients with more than 15 years of diabetic duration or islet $\beta$ cell function failure, patients with any other conditions that prevent them from undergoing surgery (including recent infections, pregnancy, coronary artery disease, cerebrovascular disease, liver or renal dysfunction, tumors, or mental disease), as well as being enrolled in another trial within the last 3 months.

\section{Data Collection and Assessment}

Patients who underwent metabolic surgery completed their evaluations both before and after the operation, and were subsequently followed up for 2 years at 3,6,12, and 24 months, respectively. Baseline clinical data were collected, including age, sex, abdominal circumference, and blood pressure. At each follow-up, some general clinical data needed to be collected repeatedly; laboratory tests were performed after an overnight fast, including measurements of fasting plasma glucose, glycosylated haemoglobin A1c (HbA1c), serum insulin, triglyceride, cholesterol, uric acid, and high-sensitivity C-reactive protein ( $h s$-CRP) levels. Visceral fat thickness (VFT) was also examined by an abdominal fat ultrasound. Homeostasis model assessment of insulin resistance (HOMA-IR) was calculated using the following equation: [(Fasting insulin (FINS, mIU/L) $\times$ Fasting plasma glucose (FPG, mmol/L))/22.5].

\section{Statistical Analysis}

The data were expressed as mean \pm SEM or SD for normally distributed variables and median (25th and 75th percentiles) for non-normally distributed variables. Comparisons between the groups in cell study were made using a twotailed unpaired Student's $t$-test or one-way ANOVA with Bonferroni's post-hoc test. Comparisons of variables before and after treatments were analysed using the Wilcoxon signed-rank matched pair test. The $\chi 2$ test was used for categorical variables. Spearman's nonparametric correlation analysis was performed to determine the relationships between $h s$-CRP changes and other factors. A two-tailed $\mathrm{p}<0.05$ was considered statistically significant, and all the results were analysed using SPSS 18.0.

\section{Results}

\section{Baseline Characteristics of the Participants}

All patients who underwent metabolic surgery were divided into non-obese $(\mathrm{BMI}<28)$ and obese $(\mathrm{BMI} \geq 28)$ groups. The baseline characteristics of the participants are presented in Table 1. Aside from BMI, obese patients had larger waist 
Table I Baseline Characteristics of the Patients Who Underwent Metabolic Surgery

\begin{tabular}{|c|c|c|}
\hline & BMI $<28(n=42)$ & $B M I \geq 28 \quad(n=85)$ \\
\hline Age (year) & $48(44-54)$ & $35(28-57)$ \\
\hline Gender (M/F) & $20 / 22$ & $34 / 51$ \\
\hline BMI $\left(\mathrm{kg} / \mathrm{m}^{2}\right)$ & $26.0(24.6-27.2)$ & $31.8(29.9-38.2) * *$ \\
\hline WC $(\mathrm{cm})$ & $90(85-94)$ & $105(98-116)^{* *}$ \\
\hline VFT $(\mathrm{cm})$ & $8.6(7.5-24)$ & $12.3(10.5-29)^{* *}$ \\
\hline SBP (mmHg) & $125(120-135)$ & $130(\mid 20-142)^{* *}$ \\
\hline $\mathrm{DBP}(\mathrm{mmHg})$ & $76(70-83)$ & $81(76-90)^{* *}$ \\
\hline$h s-C R P(\mathrm{mmol} / \mathrm{L})$ & $2.0(1.38-3.4 I)$ & $4.27(2.09-8.53)^{* *}$ \\
\hline FPG (mmol/L) & $7.5(5.7-9.6)$ & $7.0(5.5-9.5)$ \\
\hline $\mathrm{FINs}(\mathrm{mmol} / \mathrm{L})$ & $9.0(6.5-12.3)$ & $17.7(10.8-30.8)^{* *}$ \\
\hline HOMA-IR & $3.3(1.7-5.4)$ & $6.5(3.9-9.1)^{* *}$ \\
\hline HbAlc (\%) & $7.8(6.8-9.5)$ & $7.6(6.0-8.7)$ \\
\hline $\mathrm{TC}(\mathrm{mmol} / \mathrm{L})$ & $4.8(4.0-5.4)$ & $4.7(4.1-5.3)$ \\
\hline TG (mmol/L) & $1.8(1.2-2.7)$ & $2.2(1.5-3.1)$ \\
\hline HDL-c (mmol/L) & I.I (0.9-I.3) & $1.0(0.8-1.1)$ \\
\hline LDL-c (mmol/L) & $2.7(2.2-3.1)$ & $2.8(2.3-3.3)$ \\
\hline UA (umol/L) & $324.8(260.3-4 \mid 3.7)$ & $396.8(340.2-475.2) *$ \\
\hline
\end{tabular}

Notes: $* p<0.05, * * p<0.0$ l, compared with $\mathrm{BMI}<28$.

Abbreviations: BMI, body mass index (calculated as weight in kilograms divided by height in meters squared); WC, waist circumference; HbAlc, glycosylated haemoglobin; VFT, visceral fat thickness; SBP, systolic blood pressure; DBP, diastolic blood pressure; FPG, fasting plasma glucose; FINs, fasting insulins; HOMA-IR, homeostasis model assessment-insulin resistant; UA, uric acid; TC, total cholesterol; TG, triglyceride; LDL-c, low-density lipoprotein cholesterol; HDL-c, high density lipoprotein cholesterol; $h s$-CRP, high-sensitivity C-reactive protein.

circumferences (WC), higher levels of visceral fat thickness (VFT), higher uric acid levels and HOMA-IR. Importantly, a higher $h s$-CRP level was detected in obese patients, although there were similar levels of fasting glucose, $\mathrm{HbA1c}$, and lipid profiles between the two groups. Obese patients had higher systolic blood pressure [130 (120-142) versus $125(120-135) \mathrm{mm} \mathrm{Hg}$, respectively, $\mathrm{p}<0.01]$ and diastolic blood pressure [81 (76-90) versus $76(70-83) \mathrm{mm}$ $\mathrm{Hg}$, respectively, $\mathrm{p}<0.01]$ than patients with lower BMI.

\section{Effects of hs-CRP on Preadipocytes and Vascular Cells}

$h s$-CRP is well known to contribute the metabolic syndrome and atherosclerosis, but it remains elusive whether adipose tissue produces this inflammatory factor and how it affects the adipocyte and vascular cells. The adipose tissue of human mesentery was derived from the greater omentum which was removed by metabolic surgery. The use of human tissue specimen had been informed consent before operation. Immunofluorescence staining showed that CRP expressed in both in 3T3-L1 preadipocytes (Figure 1A) and in human adipose tissue, and mainly distributed in the cell membrane (Figure 1B). CRP mRNA and protein expression were also detected in human mesenteric adipose tissue (Figure 1C and D). We also showed that the administration of CRP significantly increased intracellular-free calcium concentration $\left[\mathrm{Ca}^{2+}\right]_{\mathrm{i}}$ in 3T3-L1 preadipocytes (F340/ $\mathrm{F} 3801.06+0.20, \mathrm{n}=6$ ) (Figure 1E), and in cultured VSMC (F340/F380 1.16+0.09, n=6), compared with control cells (Figure 1F). In addition, the administration of CRP dosedependently increased $\left[\mathrm{Ca}^{2+}\right]_{\mathrm{i}}$ in 3T3-L1 cells and cultured VSMC. These results suggest that CRP not only exists in adipocyte tissue but also has an impact on both adipocyte and the vascular cells.

\section{Effects of CRP on RAS/ERK Pathways in Adipocytes and Vascular Smooth Muscle Cells}

Then, we examined the effect of CRP on calcium signalling and the RAS/ERK pathways, which play a crucial role in the pathogenesis of hypertension and obesity. ${ }^{5,31}$ We showed that the administration CRP significantly increased the expression of ACE, Ang II, AT1R and p-ERK in mature 3T3-L1 adipocytes in a concentration-dependent manner, while significantly reduced the expression of ACE2 (Figure 2A). Similarly, CRP administration for $24 \mathrm{~h}$ significantly increased the expressions of ACE, Ang II and AT1R in a dose-dependent manner, and increased p-ERK expression but reduced the ACE2 expression in cultured VSMCs (Figure 2B). These results indicate that CPR has direct effects on the RAS/ERK pathways in both adipocyte tissue and vascular cells.

\section{Plasma hs-CRP Level Was Associated with Blood Pressure and Obesity}

Having found that CRP directly stimulates RAS and ERK in adipocytes and vascular cells, ${ }^{12,32}$ we then investigated whether $h s$-CRP was associated with obesity and blood pressure in patients. We showed that plasma $h s$-CRP levels were positively correlated with fat thickness (VFT), WC and SBP in patients $(\mathrm{p}<0.01)$ (Figures $3 \mathrm{~A}-\mathrm{C})$. A similar positive correlation among SBP, WC and VFT was demonstrated in patients $(\mathrm{p}<0.01)$ (Figures $3 \mathrm{D}$ and E). These results indicate that abdominal fat and CRP are highly associated with blood pressure.

\section{Effects of Metabolic Surgery on CRP and Blood Pressure}

Surgical therapy can antagonize hypertension and obesity, but its mechanism for doing so remains elusive. We aimed 

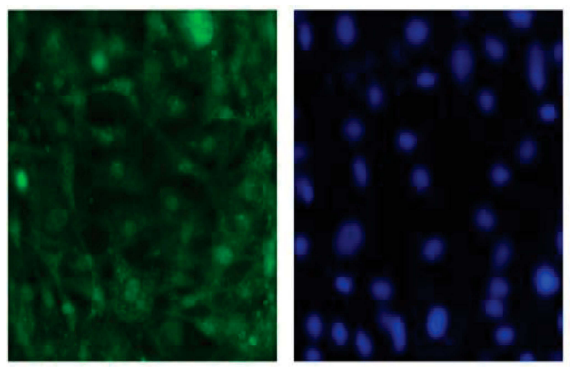

A

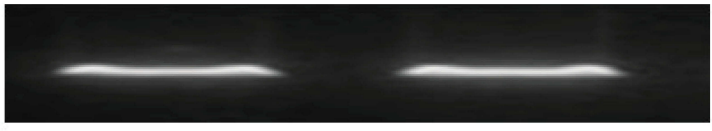

CRP

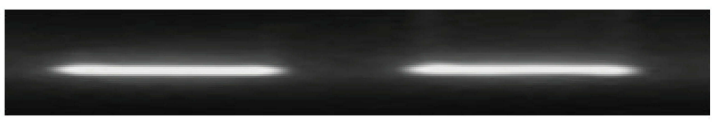

GAPDH

\section{C}
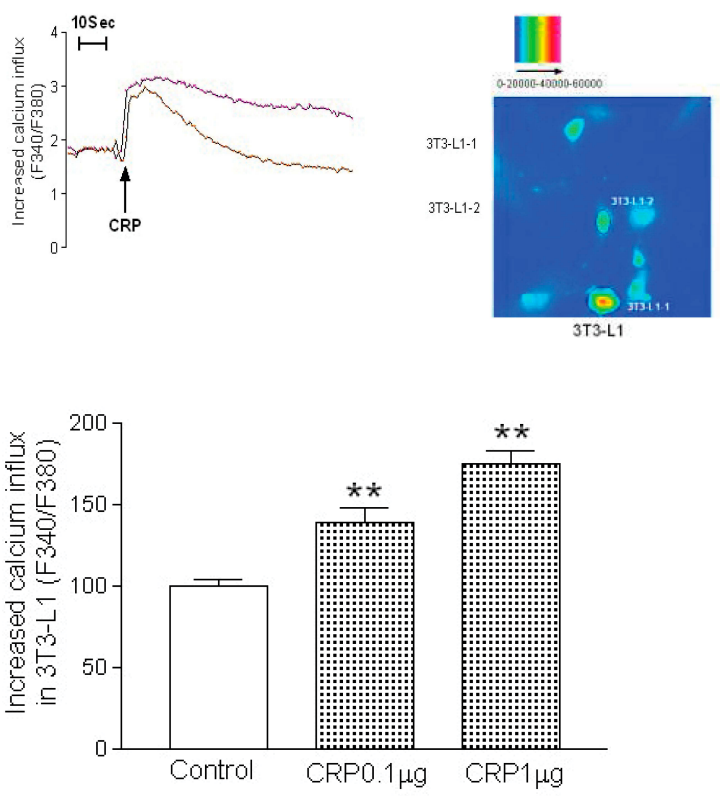

E

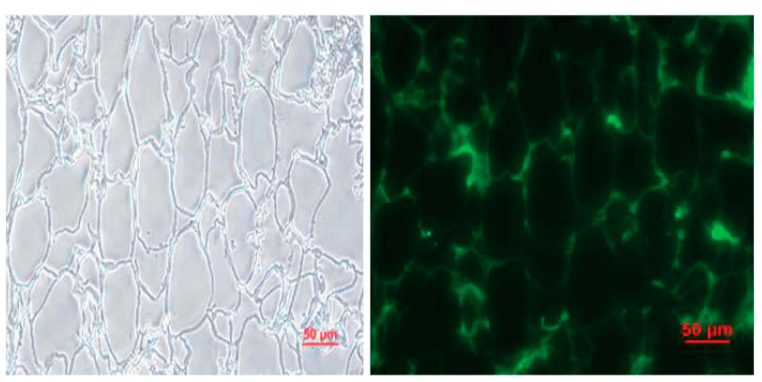

B

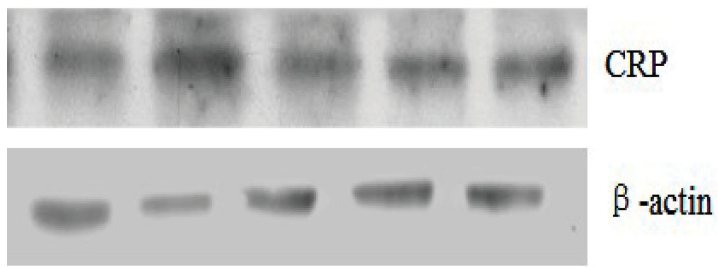

D
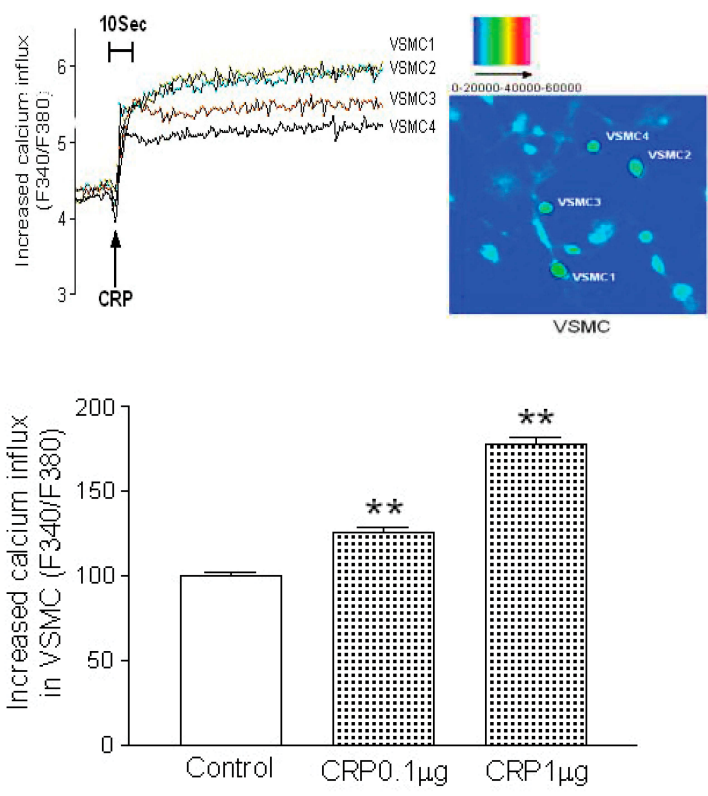

$\mathbf{F}$

Figure I CRP distribution and effects on the cytosolic calcium levels in cultured cells. Immunofluorescence staining of CRP in 3T3-LI preadipocytes (A) and adipose tissue (B), green fluorescence represents CRP distribution, while blue fluorescence represents the nucleus (A and B). Representative RT-PCR and Western blot of CRP from human mesenteric adipose tissue $(\mathbf{C}$ and $\mathbf{D})$. The quantitative results are shown below. Data are expressed as mean \pm SD. Representative traces and ratio fluorescence imaging showed the effect of CRP $(\mathrm{I} \mu \mathrm{g} / \mathrm{mL})$ on calcium $\left(\left[\mathrm{Ca}^{2+}\right]_{i}\right)$ in cultured $3 \mathrm{~T} 3-\mathrm{LI}$ preadipocytes $(\mathbf{E})$ and vascular smooth muscle cells $(\mathrm{VSMC})(\mathbf{F})$. The quantitative results and dose-dependent CRP effects on $\left[\mathrm{Ca}^{2+}\right]_{i}$ are shown ( $\mathrm{n}=6$ in independent experiments). Data are expressed as mean $\pm \mathrm{SD}$, $* * p<0.0 \mathrm{I}$ versus control.

to understand whether the antihypertensive effects of metabolic surgery were associated with the reduction of $h s$-CRP. During our long-term follow-up, we found that metabolic surgery time-dependently decreased both systolic and diastolic blood pressure (Figures 4A and B ). Additionally, a significant decrease of BP occurred at 12 months after the operation. CRP also showed a timedependent decline, with a rapid reduction at 3 months following the operation, which was consistent with the declining trend in patients' blood pressure (Figure 4C). These results suggest that the antihypertensive effects of metabolic surgery could be associated with the reduction 

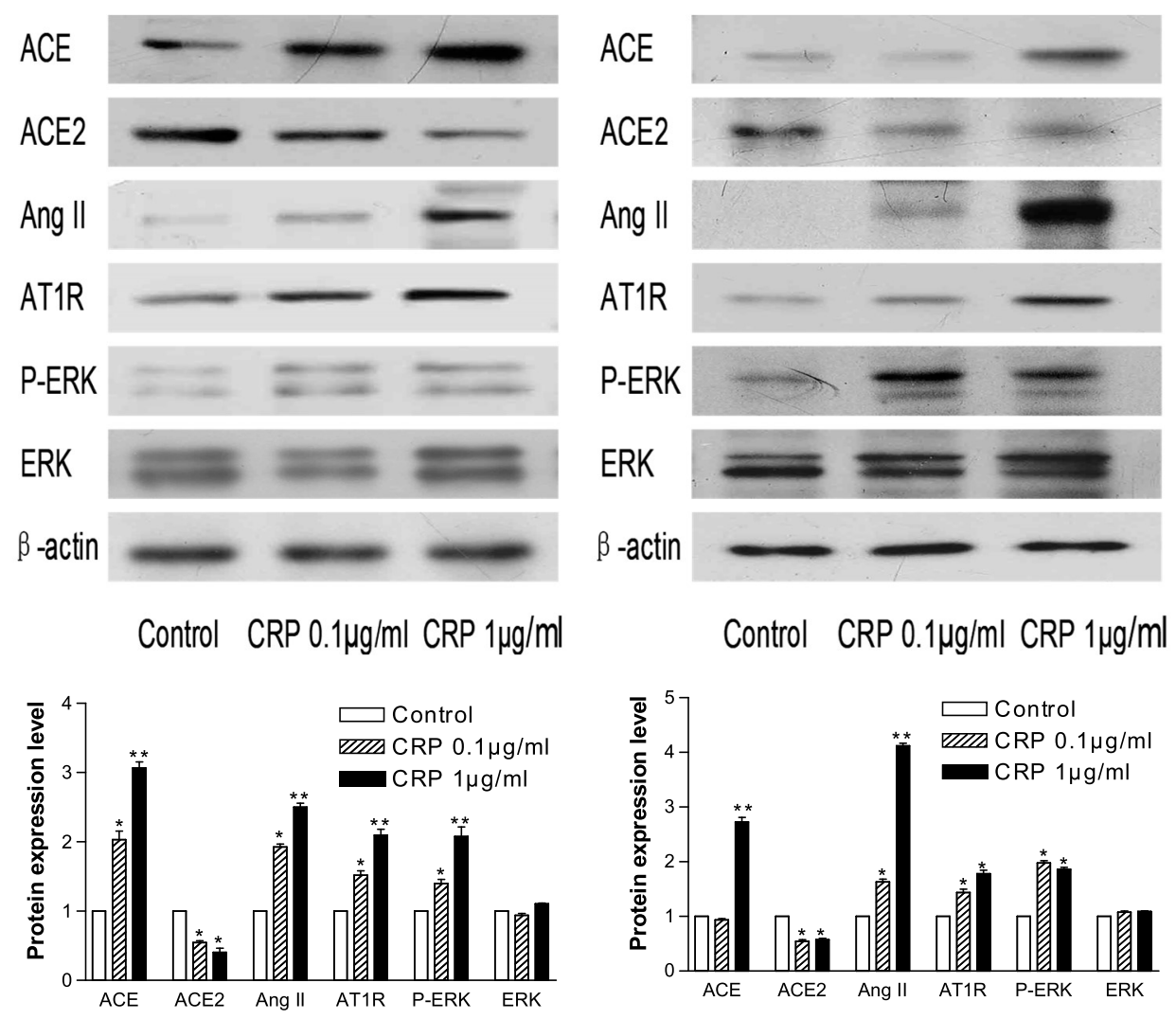

A

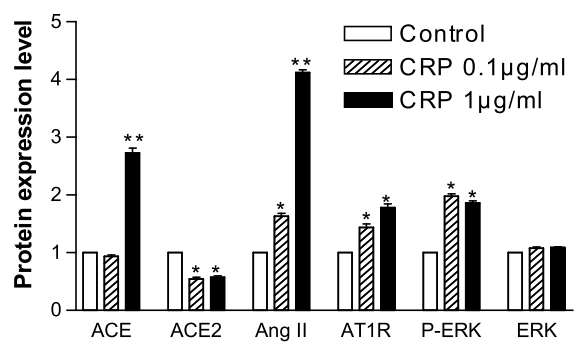

B

Figure 2 Representative Western blots of CRP on RAS/ERK pathway in 3T3-LI adipocytes (A) and VSMCs (B). The quantitative results are shown below ( $\mathrm{n}=6$ ). Data are expressed as mean $\pm \mathrm{SD},{ }^{*} p<0.05$ versus control; ${ }^{*} p<0.01$ versus control.

of the inflammatory factor related to the decrease of CRP, which thereby affected blood pressure in patients who underwent metabolic surgery.

\section{Discussion}

The major findings in this study are as follows: diabetic patients with obesity had higher blood pressure, more visceral fat accumulation and higher serum $h s$-CRP levels. Moreover, serum $h s$-CRP levels were highly correlated with blood pressure and visceral fat accumulation in patients. Adipose tissue also produced CRP, and CRP directly activated RAS and ERK in both adipose and vascular tissues. Metabolic surgery not only improved abdominal obesity and hypertension, but also lowered serum $h s$-CRP. This study highlights the fact that CRP itself contributes to the development of hypertension and fat illness in diabetic patients, which can be antagonized by surgical treatment.

It is documented that visceral adiposity is closely associated with hypertension and diabetes. ${ }^{33,34}$ Excess visceral fat accumulation causes chronic low-grade inflammation and initiates the progression of cardiovascular diseases. One study showed a significant correlation between visceral adiposity and $h s$-CRP in Japanese subjects. ${ }^{16}$ Our previous observations demonstrated that serum $h s$-CRP was associated with the component of metabolic syndrome and correlated with target organ damage in patients with metabolic syndrome. ${ }^{20}$ The present study also found that abdominal obesity was highly correlated with blood pressure and serum $h s$-CRP in obese diabetic patients. ${ }^{35}$ Although the liver is the main site of CRP production, CRP is also produced in adipose tissue. ${ }^{36}$ Additionally, adipose tissue is an active endocrine organ and secretes a variety of bioactive factors, such as adipokines, including tumor necrosis factor (TNF)- $\alpha$, monocyte chemoattractant protein (MCP)-1, and interleukin (IL)-6. All of these factors are stimulants of CRP synthesis and secretion. ${ }^{37}$ In addition to showing that CRP produced in adipose tissue, our study also demonstrated that the administration of CRP remarkably raised $\left[\mathrm{Ca}^{2+}\right]_{\mathrm{i}}$, a universal regulator of various cellular functions, in both adipocytes and VSMCs. In blood vessels, $\mathrm{Ca}^{2+}$ is the central element 
A

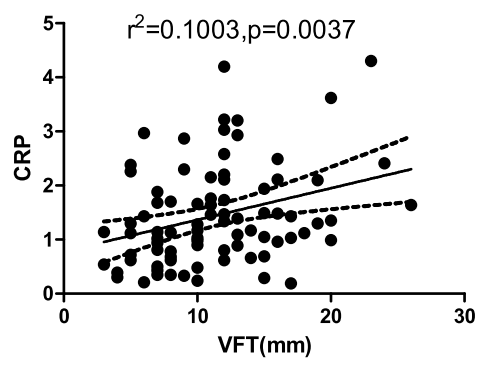

D

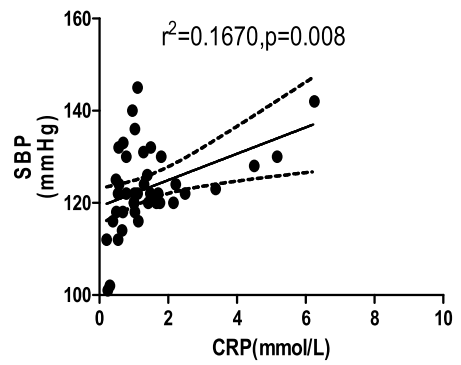

B
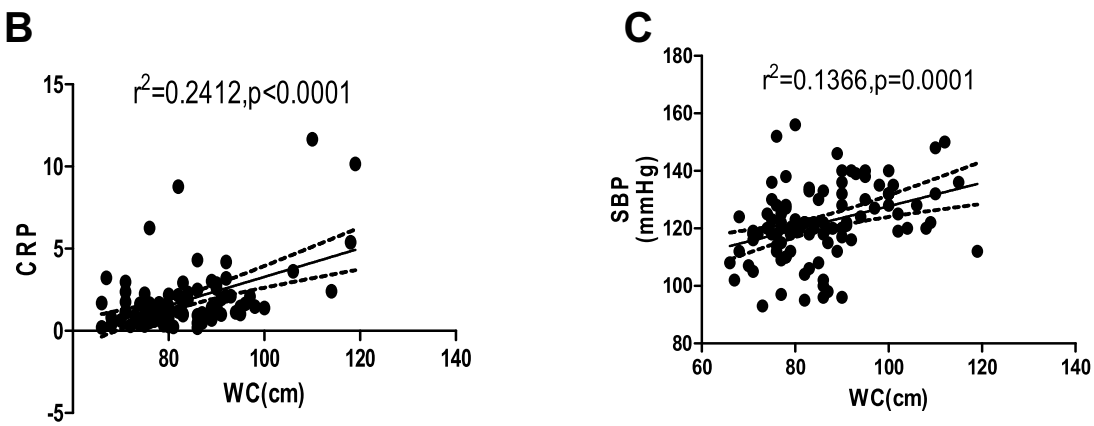

Figure 3 Analysis of the correlation among CRP, WC, VFT and SBP. (A) The correlation of CRP with VFT $\left(r^{2}=0.1003, p=0.0037, n=82\right)$. (B) The correlation of CRP with WC $\left(r^{2}=0.2412, p<0.000 I, n=87\right)$. (C) The correlation of SBP with WC $\left(r^{2}=0.1366, p=0.000 I, n=102\right)$. (D) The correlation of SBP with CRP $\left(r^{2}=0.1670, p=0.008, n=4 I\right) .(E)$ The correlation of SBP with VFT $\left(r^{2}=0.1932, p=0.0051, n=39\right)$.

A

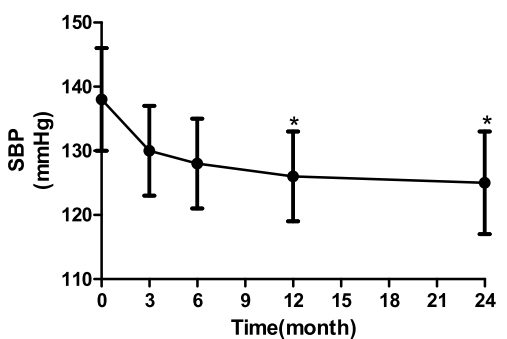

B

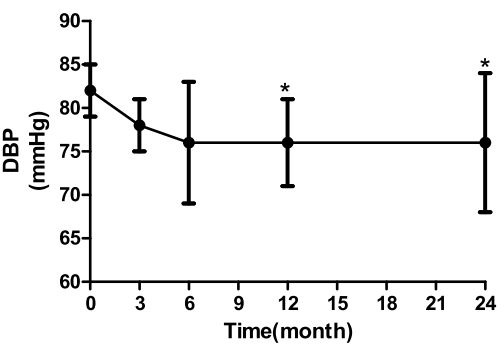

C

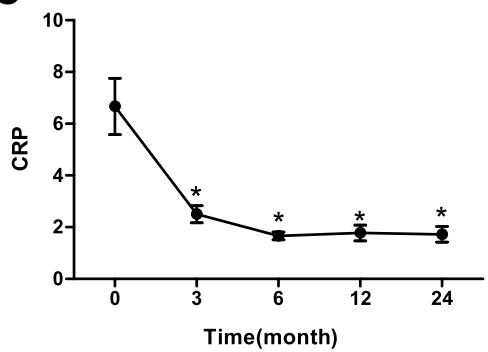

Figure 4 The effects of metabolic surgery on CRP and blood pressure. (A) The time-dependent decrease of SBP during follow-up. Data are expressed as mean \pm SD, $* p<0.05$ vs 0 month, $n=45,45,43,42$, and 40 , respectively. (B) The time-dependent decrease of DBP during follow-up. Data are expressed as mean \pm SD, $* p<0.05$ versus 0 month, $\mathrm{n}=45,45,43,42$, and 40 , respectively. (C) The time-dependent decrease of CRP during follow-up. Data are expressed as mean $\pm S D$, * $p<0.05$ versus 0 month, $n=45,45,43$, 42 , and 40 , respectively.

of excitation-contraction coupling, but it also impacts diverse signalling cascades and influences the regulation of gene expression. Disturbances in cellular $\mathrm{Ca}^{2+}$-handling and alterations in $\mathrm{Ca}^{2+}$-dependent kinase pathways are pivotal characteristics related to cardiovascular remodelling and dysfunction. This study's indication that CRP initiates a $\mathrm{Ca}^{2+}$-dependent regulatory pathway might offer broad therapeutic potential.

Although $h s$-CRP is clinically useful as a biomarker for $\mathrm{CV}$ risk prediction, some mechanistic studies suggest that CRP itself is likely involved in the pathogenesis of cardiovascular diseases. ${ }^{38}$ Procter et al identified the impact of inflammation (myeloperoxidase and CRP) and impaired nitric oxide (NO) signaling in acute and chronic atrial fibrillation (AF). ${ }^{39}$ The activation of RAS is associated with the pathogenesis of obesity and hypertension. ${ }^{40}$ Angiotensin I (Ang I) is cleaved by angiotensin-converting enzyme (ACE) to Ang II, which is metabolized by ACE2 to Ang-(1-7). Ang II binds to Ang II type 1 receptors (AT1Rs), which causes vasoconstriction, inflammation, fibrosis, and apoptosis. Reduced ACE2 shifts the balance in the RAS toward the Ang II/AT1 Raxis, resulting in the 
progression of cardiometabolic dysfunction. ${ }^{41,42}$ We showed that CRP dose-dependently increases the expressions of ACE, AngII and AT1R proteins, but inhibits ACE2 expression in both adipocytes and VSMCs, suggesting the detrimental effect of CRP on vasculatures is connected with an imbalance of RAS. Ang II/ATR1 signalling also leads to the activation of several downstream kinase pathways, such as extracellular signal-regulated kinases (ERK) $1 / 2$. . $^{43,44}$

Relatedly, $\mathrm{Ca}^{2+}$ is required for VEGF-induced ERK1/2 activation in endothelial cells. Changes in arterial $\mathrm{Ca}^{2+}$ handling in obesity links the increased activity of the ERK-MAPK signalling pathways. Several studies have reported that CRP promotes VSMC proliferation through the activation of the ERK 1/2 pathway. ${ }^{44,45}$ Our results showed that CRP significantly enhanced phosphorylated ERK expression in both adipocyte tissue and VSMCs, suggesting that CRPassociated obesity and hypertension might be related to the calcium signalling-mediated RAS and ERK pathways.

Currently, hypertension and diabetes can be well treated by pharmacotherapy. However, no optimal therapy for obesity is available. Metabolic surgery is the most effective treatment for obesity and its complications. ${ }^{46}$ In addition, clinical trials have demonstrated that metabolic surgery can effectively improve hypertension and cardiovascular events. ${ }^{47}$ However, the underlying mechanisms of metabolic surgery remain poorly understood, although metabolic surgery-mediated changes in gut hormones, nutrient sensing, intestinal glucose utilization, microbiota, and bile acid metabolism in diabetes and obesity have been proposed to function as such mechanisms. ${ }^{48-51}$ Our previous study demonstrated that metabolic surgery ameliorates hypertension through the inhibition of the activation of the sympathetic nervous system in both hypertensive patients and genetic hypertensive rats. ${ }^{52}$ Additionally, Pardina et al, found that morbidly obese patients had a greater amount of CRP in their plasma and adipose tissue compared with control subjects, and that plasma CRP can reflect decreases in inflammation and improvements with bariatric surgeryrelated weight loss. ${ }^{53}$ Our previous study showed that metformin reduced abdominal obesity and serum $h s$-CRP levels in obese hypertensive patients without diabetes. ${ }^{54}$ In this study, we further demonstrated that metabolic surgery not only lowered the blood pressure and body weight, but also reduced serum $h s$-CRP levels. This suggests that the inhibition of inflammation and its detrimental effects on cardiometabolic organs could be one of the key mechanisms underlying the success of metabolic surgery.

\section{Conclusions}

In conclusion, our study demonstrates that CRP is highly correlated with hypertension and visceral adiposity. CRP is not only a biomarker, but is also a participating factor in the pathogenesis of obesity and hypertension, where it functions through impacting the RAS and ERK pathways. Our findings can, therefore, be understood as providing key insights into the beneficial effects of metabolic surgery, which, as we show, could be partly associated with antagonizing inflammation. Thus, metabolic surgery may represent a promising intervention in obese hypertensive patients with diabetes.

\section{Abbreviations}

BMI, body mass index; WC, waist circumference; HbA1c, glycosylated haemoglobin; VFT, visceral fat thickness; SBP, systolic blood pressure; DBP, diastolic blood pressure; FPG, fasting plasma glucose; FINs, fasting insulins; HOMA-IR, homeostasis model assessment-insulin resistant; UA, uric acid; TC, total cholesterol; TG, triglyceride; LDL-c, low-density lipoprotein cholesterol; HDL-c, highdensity lipoprotein cholesterol; $h s-\mathrm{CRP}$, high-sensitivity C-reactive protein.

\section{Data Sharing Statement}

All data generated or analyzed during this study are included in this published article and information about experimental sessions and results are available from the corresponding author on reasonable request.

\section{Ethics and Consent Statement}

The animal experiments were performed in compliance with and were approved by the ethics committee of the Daping Hospital, the Third Military Medical University. All experiments were performed following institutional guidelines and regulations of ethics committee of the Daping Hospital, the Third Military Medical University. All patients provided written informed consent.

\section{Acknowledgments}

We thank Lijuan Wang and Tingbing Cao for their technical assistance. Fang Sun and Zhigang Zhao are co-first authors for this study.

\section{Author Contributions}

All authors made substantial contributions to conception and design, acquisition of data, or analysis and 
interpretation of data; took part in drafting the article or revising it critically for important intellectual content; gave final approval of the version to be published; and agree to be accountable for all aspects of the work.

\section{Funding}

This study was supported by grants from the National Key Research and Development Project (2018YFA0800601) and National Natural Science Foundation of China (81470536, 81721001, 81900380). The funders had no role in the design and conduct of the study; collection, management, analysis, and interpretation of the data; and preparation, review, or approval of the manuscript.

\section{Disclosure}

The authors declared that they have no conflicts of Financial interests/non-financial interests in this work.

\section{References}

1. Whelton PK, Carey RM, Aronow WS, et al. 2017 ACC/AHA/AAPA/ $\mathrm{ABC} / \mathrm{ACPM} / \mathrm{AGS} / \mathrm{APhA} / \mathrm{ASH} / \mathrm{ASPC} / \mathrm{NMA} / \mathrm{PCNA}$ guideline for the prevention, detection, evaluation, and management of high blood pressure in adults: executive summary: a report of the American College of Cardiology/American Heart Association Task Force on Clinical Practice Guidelines. Circulation. 2018;138(17):e426-e483. doi:10.1161/CIR.0000000000000597

2. Lastra G, Syed S, Kurukulasuriya LR, Manrique C, Sowers JR. Type 2 diabetes mellitus and hypertension: an update. Endocrinol Metab Clin North Am. 2014;43(1):103-122. doi:10.1016/j.ecl.2013.09.005

3. Van Gaal LF, Mertens IL, De Block CE. Mechanisms linking obesity with cardiovascular disease. Nature. 2006;444(7121):875-880. doi:10.1038/nature05487

4. American Diabetes Association. 9. Cardiovascular disease and risk management: standards of medical care in Diabetes-2018. Diabetes Care. 2018;41(Suppl Supplement 1):S86-S104. doi:10.2337/dc18-S009

5. Cabandugama PK, Gardner MJ, Sowers JR. The renin angiotensin aldosterone system in obesity and hypertension: roles in the cardiorenal metabolic syndrome. Med Clin North Am. 2017;101(1):129-137. doi:10.1016/j.mcna.2016.08.009

6. Hevener AL, Febbraio MA, Group tSCW. The 2009 stock conference report: inflammation, obesity and metabolic disease. Obes Rev 2010;11(9):635-644. doi:10.1111/j.1467-789X.2009.00691.x

7. Saxton SN, Clark BJ, Withers SB, Eringa EC, Heagerty AM. Mechanistic links between obesity, diabetes, and blood pressure: role of perivascular adipose tissue. Physiol Rev. 2019;99 (4):1701-1763. doi:10.1152/physrev.00034.2018

8. Dalmas E, Venteclef N, Caer C, et al. T cell-derived IL-22 amplifies IL-1beta-driven inflammation in human adipose tissue: relevance to obesity and type 2 diabetes. Diabetes. 2014;63(6):1966-1977. doi: $10.2337 / \mathrm{db} 13-1511$

9. Westover AJ, Johnston KA. An immunomodulatory device improves insulin resistance in obese porcine model of metabolic syndrome. J Diabetes res. 2016;2016:3486727.

10. Deraz TE, Kamel TB, El-Kerdany TA, El-Ghazoly HMA. Highsensitivity $\mathrm{C}$ reactive protein as a biomarker for grading of childhood asthma in relation to clinical classification, induced sputum cellularity, and spirometry. Pediatr Pulmonol. 2012;47(3):220-225. doi:10.1002/ppul.21539
11. Roh EJ, Lim JW, Ko KO, Cheon EJ. A useful predictor of early atherosclerosis in obese children: serum high-sensitivity C-reactive protein. J Korean Med Sci. 2017;22(2):192-197.

12. Ouchi N, Kihara S, Funahashi T, Nakamura T, Matsuzawa Y. Reciprocal association of C-Reactive protein with adiponectin in blood stream and adipose tissue. Circulation. 2003;107(5):671-674. doi:10.1161/01.CIR.0000055188.83694.B3

13. Rossi AP, Fantin F, Zamboni GA, Mazzali G, Zamboni M. Predictors of ectopic fat accumulation in liver and pancreas in obese men and women. Obesity. 2011;19(9):1747-1754. doi:10.1038/oby.2011.114

14. Bataille R, Klein B. C-reactive protein levels as a direct indicator of interleukin-6 levels in humans in vivo. Arthritis Rheum. 2010;35 (8):982-983.

15. Yap S, Moshage H, Hazenberg B, et al. Tumor necrosis factor (TNF) inhibits interleukin (IL)-1 and/or IL-6 stimulated synthesis of C-reactive protein (CRP) and serum amyloid A (SAA) in primary cultures of human hepatocytes. J Hepatol. 1989;9(SUPPL. 1):S97. doi:10.1016/0168-8278(89)90370-X

16. Tsuriya D, Morita H, Morioka T, et al. Significant correlation between visceral adiposity and high-sensitivity C-reactive protein (hs-CRP) in Japanese subjects. Internal Med. 2011;50(22):2767-2773. doi:10.2169/ internalmedicine. 50.5908

17. Visser M. Elevated C-Reactive protein levels in overweight and obese adults. JAMA. 1999;282(22):2131-2135.

18. Fonseca FA, Izar I. High-sensitivity C-Reactive protein and cardiovascular disease across countries and ethnicities. Clinics. 2016;71 (4):235-242. doi:10.6061/clinics/2016(04)11

19. Laaksonen DE, Niskanen L, Nyyssönen K, et al. C-reactive protein and the development of the metabolic syndrome and diabetes in middle-aged men. Diabetologia. 2004;47(8):1403-1410.

20. Zhao Z, Nie H, He H, et al. High-sensitivity C-reactive protein predicts target organ damage in Chinese patients with metabolic syndrome. Metabolism. 2007;56(12):1612-1619.

21. Han TS, Sattar N, Williams K, Gonzalez-Villalpando C, Lean MEJ, Haffner SM. Prospective study of C-Reactive protein in relation to the development of diabetes and metabolic syndrome in the Mexico City diabetes study. Diabetes care. 2002;25(11):2016-2021.

22. Anty R, Bekri S, Luciani N, et al. The inflammatory C-Reactive protein is increased in both liver and adipose tissue in severely obese patients independently from metabolic syndrome, type 2 diabetes, and NASH. Am J Gastroenterol. 2006;101(8):1824-1833. doi:10.1111/j.1572-0241.2006.00724.x

23. Bekri S, Gual P, Anty R, et al. Increased adipose tissue expression of hepcidin in severe obesity is independent from diabetes and NASH. Gastroenterology. 2006;131(3):788-796. doi:10.1053/j.gastro.2006.07. 007

24. Huang Y, Noble NA, Zhang J, Xu C, Border WA. Renin-stimulated TGF$\beta 1$ expression is regulated by a mitogen-activated protein kinase in mesangial cells. Kidney Int. 2007;72(1):45-52. doi:10.1038/sj.ki.5002243

25. Chitturi S, George J. Hepatotoxicity of commonly used drugs: nonsteroidal anti-inflammatory drugs, antihypertensives, antidiabetic agents, anticonvulsants, lipid-lowering agents, psychotropic drugs. Semin Liver Dis. 2002;22(02):169-184.

26. Genser L, Barrat C. Bariatric surgery versus intensive medical therapy for diabetes -5- year outcomes. Obésité. 2017;12(1):65-67. doi:10.1007/s11690-017-0567-8

27. Jirapinyo P, Haas AV, Thompson CC. Effect of the duodenal-jejunal bypass liner on glycemic control in patients with type 2 diabetes with obesity: a meta-analysis with secondary analysis on weight loss and hormonal changes. Diabetes Care. 2018;41(5):1106. doi:10.2337/ dc17-1985

28. Rossi G, Association AD. Diagnosis and classification of diabetes mellitus. Diabetes Care. 2018;33(Supp1 1):S62-S69.

29. Ioannidis JPA. Diagnosis and treatment of hypertension in the 2017 ACC/AHA guidelines and in the real world. JAMA. 2018;319(2):115. doi:10.1001/jama.2017.19672 
30. Alberti G, Zimmet P, Shaw J, Grundy SM. The IDF consensus worldwide definition of the metabolic syndrome. 2006.

31. Nakashima H, Miho N, Ishida M, et al. 4P-1129 role of Ras-ERK pathway and $\mathrm{c}$-Src in angiotensin II-induced osteopontin expression in vascular smooth muscle cells. Atheroscler Suppl. 2003;4(2):323. doi:10.1016/S1567-5688(03)91385-7

32. Han C, Liu J, Liu X, Li M. Angiotensin II induces C-reactive protein expression through ERK1/2 and JNK signaling in human aortic endothelial cells. Atherosclerosis. 2010;212(1):206-212. doi:10.10 16/j.atherosclerosis.2010.05.020

33. Koh H, Hayashi T, Sato KK, et al. Visceral adiposity, not abdominal subcutaneous fat area, is associated with high blood pressure in Japanese men: the Ohtori study. Hypertens Res. 2011;34(5):565. doi: $10.1038 / \mathrm{hr} .2010 .271$

34. Lv X Zhou W, Sun J, et al.Visceral adiposity is significantly associated with type 2 diabetes in middle-aged and elderly Chinese women: a cross-sectional study. J Diabetes. 2016;9(10):920-928. doi:10.1111/1753-0407.12499

35. Uemura H, Katsuura-Kamano S, Yamaguchi M, Bahari T, Arisawa K. Relationships of serum high-sensitivity C-reactive protein and body size with insulin resistance in a Japanese cohort. PLoS One. 2017;12 (6):e0178672. doi:10.1371/journal.pone.0178672

36. Sanchez-Infantes D, Stephens JM. Role of Adipose Tissue in the Pathogenesis and Treatment of Metabolic Syndrome. Springer International Publishing; 2014.

37. Hao WR, Li-Chin S, Chun-Chao C, et al. Cafestol inhibits cyclic-straininduced interleukin-8, intercellular adhesion molecule-1, and monocyte chemoattractant protein-1 production in vascular endothelial cells. Oxid Med Cell Longev. 2018;2018:1-10. doi:10.1155/2018/7861518

38. Larsen SB, Grove EL, Wurtz M, Neergaard-Petersen S, Hvas AM, Kristensen SD. The influence of low-grade inflammation on platelets in patients with stable coronary artery disease. Thromb Haemost. 2015;114(3):519-529. doi:10.1160/TH14-12-1007

39. Procter NE, Ball J, Ngo DT, et al. Platelet hyperaggregability in patients with atrial fibrillation. Evidence of a background proinflammatory milieu. Herz. 2016;41(1):57-62. doi:10.1007/s00059-015-4335-y

40. Quadri SS, Culver S, Ramkumar N, Kohan DE, Siragy HM. (Pro) Renin receptor mediates obesity-induced antinatriuresis and elevated blood pressure via upregulation of the renal epithelial sodium channel. PloS one. 2018;13(8):e0202419.

41. Eriksson U, Danilczyk U, Penninger JM. Just the beginning: novel functions for angiotensin-converting enzymes. Curr Biol. 2002;12 (21):R745-R752. doi:10.1016/S0960-9822(02)01255-1

42. Yang C-W, Lu L-C, Chang -C-C, et al. Imbalanced plasma ACE and ACE2 level in the uremic patients with cardiovascular diseases and its change during a single hemodialysis session. Ren Fail. 2017;39 (1):719-728. doi:10.1080/0886022X.2017.1398665
43. Zhong J, Zhu ZM, Yang YJ. Inhibition of PKC and ERK1/2 in cultured rat vascular smooth muscle cells by angiotensin-(1-7). Acta Physiol Sin. 2001;53(5):361-363.

44. Xie J-Y, Chen N, Ren H, Wang W-M. Angiotensin II-mediated activation of fibrotic pathways through ERK1/2 in rat peritoneal mesothelial cells. Ren Fail. 2010;32(7):871-879. doi:10.3109/ 0886022X.2010.494807

45. Pei WN, Hu HJ, Liu F, Xiao B, Zuo YB, Cui W. C-reactive protein aggravates myocardial ischemia/reperfusion injury through activation of extracellular-signal-regulated kinase 1/2. J Geriatr Cardiol. 2018;15(7):492-503. doi:10.11909/j.issn.1671-5411.2018.07.001

46. Nguyen NT, Varela JE. Bariatric surgery for obesity and metabolic disorders: state of the art. Nat Rev Gastroenterol Hepatol. 2017;14 (3):160-169. doi:10.1038/nrgastro.2016.170

47. Appachi S, Kashyap SR. 'Adiposopathy' and cardiovascular disease: the benefits of bariatric surgery. Curr Opin Cardiol. 2013;28 (5):540-546. doi:10.1097/HCO.0b013e3283642a33

48. Mazidi M, de Caravatto PP, Speakman JR, Cohen RV. Mechanisms of action of surgical interventions on weight-related diseases: the potential role of bile acids. Obes Surg. 2017;27(3):826-836. doi:10.1007/ s11695-017-2549-1

49. Andrew CA, Umashanker D, Aronne LJ, Shukla AP. Intestinal and gastric origins for diabetes resolution after bariatric surgery. Curr Obes Rep. 2018;7(2):139-146. doi:10.1007/s13679-018-0302-2

50. Hage MP, Safadi B, Salti I, Nasrallah M. Role of gut-related peptides and other hormones in the amelioration of type 2 diabetes after Roux-en-Y gastric bypass surgery. ISRN Endocrinol. 2012;2012:504756. doi:10.54 02/2012/504756

51. Lu Z, Wei X, Sun F, et al. Non-insulin determinant pathways maintain glucose homeostasis upon metabolic surgery. Cell Discovery. 2018;4(1):1-6.

52. Zhang H, Pu Y, Chen J, et al. Gastrointestinal intervention ameliorates high blood pressure through antagonizing overdrive of the sympathetic nerve in hypertensive patients and rats. $J$ Am Heart Assoc. 2014;3(5):e000929. doi:10.1161/JAHA.114.000929

53. Pardina E, Ferrer R, Baena-Fustegueras JA, et al. Only C-reactive protein, but not TNF-alpha or IL6, reflects the improvement in inflammation after bariatric surgery. Obesity surgery. 2012;22 (1):131-139.

54. He H, Zhao Z, Chen J, et al. Metformin-based treatment for obesity-related hypertension: a randomized, double-blind, placebo-controlled trial. J Hypertens. 2012;30(7):1430-1439. doi:10.1097/HJH.0b013e328353e 249

Diabetes, Metabolic Syndrome and Obesity: Targets and Therapy

Dovepress

\section{Publish your work in this journal}

Diabetes, Metabolic Syndrome and Obesity: Targets and Therapy is an international, peer-reviewed open-access journal committed to the rapid publication of the latest laboratory and clinical findings in the fields of diabetes, metabolic syndrome and obesity research. Original research, review, case reports, hypothesis formation, expert opinion and commentaries are all considered for publication. The manuscript management system is completely online and includes a very quick and fair peer-review system, which is all easy to use. Visit http://www.dovepress.com/testimonials.php to read real quotes from published authors. 